\title{
LaPT2 Gene Encodes a Flavonoid Prenyltransferase in White Lupin
}

\author{
Jinyue Liu ${ }^{1,2,3 \dagger}$, Yaying Xia ${ }^{1,2,3 \dagger}$, Wenbo Jiang ${ }^{1}$, Guoan Shen ${ }^{4}$ and Yongzhen Pang ${ }^{1 *}$ \\ ${ }^{1}$ Institute of Animal Science, Chinese Academy of Agricultural Sciences, Beijing, China, ${ }^{2}$ Key Laboratory of Plant Resources \\ and Beijing Botanical Garden, Institute of Botany, Chinese Academy of Sciences, Beijing, China, ${ }^{3}$ University of Chinese \\ Academy of Sciences, Beijing, China, ${ }^{4}$ The Institute of Medicinal Plant Development, Beijing, China
}

\section{OPEN ACCESS}

Edited by:

Deyu Xie,

North Carolina State University,

United States

Reviewed by:

Tetsuya Mori,

RIKEN Center for Sustainable Resource Science (CSRS), Japan Michał Książkiewicz, Institute of Plant Genetics, Polish Academy of Sciences (PAN), Poland

*Correspondence: Yongzhen Pang pangyongzhen@caas.cn

${ }^{+}$These authors have contributed equally to this work

Specialty section:

This article was submitted to

Plant Metabolism and

Chemodiversity,

a section of the journal

Frontiers in Plant Science

Received: 27 February 2021

Accepted: 17 May 2021

Published: 11 June 2021

Citation:

Liu J, Xia Y, Jiang W, Shen G and Pang Y (2021) LaPT2 Gene Encodes

a Flavonoid Prenyltransferase in

White Lupin.

Front. Plant Sci. 12:673337.

doi: 10.3389/fp/s.2021.673337
Legume plants are rich in prenylated flavonoid compounds, which play an important role in plant defense and human health. In the present study, we identified a prenyltransferase (PT) gene, named LaPT2, in white lupin (Lupinus albus), which shows a high identity and close relationship with the other known PT genes involved in flavonoid prenylation in planta. The recombinant LaPT2 protein expressed in yeast cells exhibited a relatively strong activity toward several flavonols (e.g., kaempferol, quercetin, and myricetin) and a relatively weak activity toward flavanone (naringenin). In addition, the recombinant LaPT2 protein was also active toward several other types of flavonoids, including galangin, morin, 5-deoxyquercetin, 4'-O-methylkaempferol, taxifolin, and aromadendrin, with distinct enzymatic affinities. The LaPT2 gene was preferentially expressed in the roots, which is consistent with the presence of prenylated flavonoid kaempferol in the roots. Moreover, we found that the expression level of $L a P T 2$ paralleled with those of $L a F 3 H 1$ and $L a F L S 2$ genes that were relatively higher in roots and lower in leaves, suggesting that they were essential for the accumulation of prenylated flavonoid kaempferol in roots. The deduced full-length LaPT2 protein and its signal peptide fused with a green fluorescent protein (GFP) are targeted to plastids in the Arabidopsis thaliana protoplast. Our study demonstrated that LaPT2 from white lupin is responsible for the biosynthesis of prenylated flavonoids, in particular flavonols, which could be utilized as phytoalexin for plant defense and bioactive flavonoid compounds for human health.

Keywords: white lupin, flavonoids, prenyltransferase, LaPT2, kaempferol prenylation

\section{INTRODUCTION}

Flavonoids, having a typical C6-C3-C6 skeleton structure, comprise one of the largest metabolite groups in the plant kingdom. Flavonoids are divided into a variety of classes; including flavones, flavonols, and isoflavonoids, depending on the levels of oxidation and the pattern of substitutions on their C ring (Middleton, 1998). These compounds possess very important physiological functions in plant growth and development and environment interaction (Dixon and Paiva, 1995).

The molecular basis for the formation of the different flavonoid skeletons has been welldocumented, and the corresponding coding genes have been identified from various plant species, especially in model plants, such as Arabidopsis thaliana, Zea mays, and Petunia hybrida (WinkelShirley, 2001). Although the number of flavonoid skeletons is limited, a great number of diverse flavonoid compounds are subsequently synthesized by using a variety of modifications, including 
glycosylation, acylation, methylation, and prenylation (Dixon and Pasinetti, 2010). Prenylation is one of the specific modification forms of flavonoids, and prenylated flavonoids were widely presented in several plant families, including Leguminosae, Moraceae, Umbelliferae, and Euphorbiaceae (Tahara and Ibrahim, 1995; Barron and Ibrahim, 1996; Boland and Donnelly, 1998; Botta et al., 2005, 2009).

Prenylated flavonoids display important physiological functions in response to environmental stress (Sohn et al., 2004). In addition, prenylated flavonoids also possess considerable health-promoting properties for human health (De Naeyer et al., 2004; Ahmed-Belkacem et al., 2005; Han et al., 2006). In particular, prenylated flavonols, such as the methylated 8-prenylkaempferol (also called sophoflavescenol), showed cytotoxicity against the different cancer cell lines as well as antidiabetic and anti-Alzheimer activities (Jung et al., 2011; Boozari et al., 2019).

Prenylation of flavonoid compounds was catalyzed by a prenyltransferase (PT) from the plant microsomal fraction (Yamamoto et al., 2000; Zhao et al., 2003). Several genes encoding flavonoid PTs have been isolated and characterized from various plant species, in particular legume plants, including SfN8DT, SfG6DT, and SfiLDT from Sophora flavescens (Sasaki et al., 2008, 2011; Chen et al., 2013), GmG4DT and GmG2DT from Glycine max (Akashi et al., 2009; Yoneyama et al., 2016; Sukumaran et al., 2018), LjG6DT from Lotus japonicus (Liu et al., 2018), and GuA6DT and GuILDT from Glycyrrhiza uralensis ( $\mathrm{Li}$ et al., 2014, 2018). Their recombinant proteins expressed in yeast microsomal fractions could utilize the different flavonoid aglycones as an acceptor, and dimethylallyl pyrophosphate (DMAPP) as a donor to introduce prenyl moiety at C6, C8, or C3' positions of specific flavonoids. However, in all these cases, none of the PTs could catalyze the prenylation of flavonols, a large group of bioactive flavonoids in planta.

White lupin (Lupinus albus) is a widely cultivated legume plant with an excellent and a high content of proteins in seeds, which was first domesticated in the Mediterranean region and was planted in many countries in the world (Lucas et al., 2015). White lupin accumulates various prenylated flavonoid compounds, and both the structure and physiological property of these flavonoids have been well-characterized (Tahara et al., 1984; Gagnon et al., 1992; Katagiri et al., 2000; Bednarek et al., 2001). However, reports on PT genes in the flavonoid pathway are still rare in white lupin. In a previous study, we identified an LaPT1 gene from white lupin, and the encoding PT protein could prenylate genistein at C3' position to produce isowighteone (Shen et al., 2012), but additional genes encoding PT that could prenylate various flavonoids remain unknown in white lupin.

In order to identify additional PT genes in this prenylflavonoidrich plant species, we further searched the white lupin transcriptome database and isolated another PT gene of LaPT2. In vitro enzymatic assays showed that the recombinant LaPT2 protein could use several different flavonoid compounds as acceptors, and DMAPP as a donor to generate prenylated flavonoids. In addition, we found that the presence of prenylkaempferol in roots is consistent with a relatively high expression level of the LaPT2 gene in the roots of white lupin. The discovery of the LaPT2 gene could provide a valuable reference for the in-depth investigation of the functional diversification and evolution of PT in planta, and metabolic engineering of bioactive prenylated flavonoids as medicines for human health.

\section{MATERIALS AND METHODS}

\section{Plant Materials and Growth Conditions}

White lupin seeds (L.albus) were obtained from the United States Department of Agriculture Soybean Germplasm Collection. The seeds were germinated and grown in vermiculite under the controlled condition of $16 / 8 \mathrm{~h}$ of light/dark cycles at $25^{\circ} \mathrm{C}$. The leaves and roots of 30-day-old were harvested separately and stored at $-80^{\circ} \mathrm{C}$ until further analysis.

\section{Chemicals}

Flavonoid standards, such as genistein, 2'-hydroxygenistein, daidzein, apigenin, kaempferol, 3'-methylkaempferol, quercetin, myricetin, 3-hydroxyflavone, naringenin, liquiritigenin, and isoliquiritigenin, were purchased from Shanghai Tongtian Biotechnology Company Ltd. (Shanghai, China), and catechin, 5-deoxyquercetin, galangin, morin, and kaempferol-3-O-glucoside were obtained from Chemfaces Biochemical Co. Ltd. (Wuhan, China), and 8-prenylated kaempferol was obtained from Yuanye Biotechnology Company Ltd. (Shanghai, China). DMAPP, farnesyl diphosphate (FPP), and geranyl diphosphate (GPP) were purchased from Sigma-Aldrich (St. Louis, MO, United States).

\section{Phylogenetic Analysis of Plant PTs}

The protein sequences of PTs involved in the natural product biosynthesis were used for the sequence alignment and construction of the phylogenetic tree. The multi-sequence alignment was performed by using Clustal X2 (Larkin et al., 2007). Then, the molecular phylogenetic tree based on the Maximum Likelihood Method was reconstructed by using the MEGA6 software with a bootstrap value of 1,000 (Tamura et al., 2013).

\section{In vitro PT Activity Assays}

The construction of a vector pDR196GW-LaPT2, yeast transformation in the W303A1 strain, and microsomal protein extraction were the same as previously reported (Liu et al., 2018). In brief, the total microsomal protein was quantified by using the Bradford (1976) assay and immediately used for PT activity assays. The enzymatic reaction contained $1 \mathrm{mM}$ of dithiothreitol, $25 \mathrm{mM}$ of MOPS ( $\mathrm{pH} \mathrm{7.0),} 10 \mathrm{mM}$ of $\mathrm{Mg}^{2+}$, $100 \mu \mathrm{M}$ of flavonoid substrates, and $20 \mu \mathrm{g}$ of total microsomal protein. The reaction mixture of $200 \mu \mathrm{l}$ was incubated at $30^{\circ} \mathrm{C}$ for $30 \mathrm{~min}$ and then terminated by the addition of $200 \mu \mathrm{l}$ of methanol. The apparent $\mathrm{Km}$ values for flavonoid substrates were determined with various concentrations of flavonoid substrates (ranging from 5 to $400 \mu \mathrm{M}$ ), $10 \mu \mathrm{g}$ of the total 
microsomal protein with a final volume of $50 \mu \mathrm{l}$. The apparent $\mathrm{Km}$ values for DMAPP (ranging from 50 to $400 \mu \mathrm{M}$ ) were determined by using $400 \mu \mathrm{M}$ kaempferol. The mixtures were incubated at $30^{\circ} \mathrm{C}$ for $30 \mathrm{~min}$, and the products were quantified by using the standard curve of corresponding flavonoid substrates through a peak area of the UV spectrum on high-performance liquid chromatography (HPLC). All reactions were performed in triplicates. The apparent $\mathrm{Km}$ values were calculated by using the Eadie-Hofstee Plot. The conversion rates (\%) were calculated as follows: peak area for a product/peak area for a substrate in control reactions.

\section{Subcellular Localization of LaPT2 Protein}

The open reading frame of LaPT2 lacking the stop codon, the 87-amino-acid transit peptide fragment, and the fragment lacking the 87-amino-acid were, respectively, subcloned into the pJIT163-green fluorescent protein (GFP) vector, resulting in the LaPT2-GFP, LaPT2-TP $1-87$-GFP, and LaPT2 ${ }_{88-403}-\mathrm{GFP}$ fusion gene under the control of a double 35S CaMV promoter. These three plasmids were further confirmed by sequencing and transforming into the Arabidopsis protoplasts via the polyethylene glycol (PEG)-mediated transformation as previously described (Yoo et al., 2007). After $16 \mathrm{~h}$ of incubation at $25^{\circ} \mathrm{C}$, the GFP fluorescence was observed under a confocal laser scanning microscope (Leica TCS SP5, Germany).

\section{Gene Expression Analysis by Quantitative Real-Time PCR}

For the analysis of gene expression in different organs, leaves and roots were separately harvested from 30-day-old white lupin plants grown in vermiculite. The collected samples were immediately frozen in liquid nitrogen and grounded into a fine powder. Total RNAs were extracted by using RNAiso Plus (code No. 9108, Takara, Japan) according to the instructions of the manufacturer. Total RNAs were treated with DNase I to digest genomic DNA and were then used as a template to synthesize the first-strand complementary DNA (cDNA) by using reverse transcriptase moloney murine leukemia virus (M-MLV; RNase H-, code No. 2641A, Takara, Japan). The cDNA was used as a template for the quantitative real-time PCR (qRT-PCR) analysis with the SYBR Green PCR kit (Kangwei Biotech, Beijing, China). The qRT-PCR analyses were performed by using gene-specific primers (Supplementary Table 2). The transcript levels of each gene were determined by relative quantification using the $2^{-\Delta \Delta \mathrm{Ct}}$ method and normalized by using the actin gene as a reference. The data were indicated as an average of biological and technical triplicates. Further, the lowest expression level of a gene in each panel (Figure 1) was set at 1 , and the data were indicated as an average of three replicates.

\section{Analysis of Flavonoid Metabolites}

For the analysis of flavonoid compounds, leaf and root materials (about $1 \mathrm{~g}$ fresh weight) were harvested and lyophilized; $20 \mathrm{mg}$ of the sample (dry weight) was extracted with $1 \mathrm{ml}$ of $80 \%$ methanol. An analysis of $20 \mu \mathrm{l}$ extracts was performed on HPLC of Agilent 1260 Infinity II (Santa Clara, CA, United States) with a linear eluting gradient $(5-70 \%$ solvent $\mathrm{B}$ over $30 \mathrm{~min}$, $70-100 \%$ solvent B in $30-35 \mathrm{~min})$ with solvent A ( $0.1 \%$ formic acid in water) and solvent $\mathrm{B}$ ( $0.1 \%$ formic acid in acetonitrile) at the flow rate of $1 \mathrm{ml} / \mathrm{min}$, with Eclipse XDB-C18 reversephase column $(4.6 \mathrm{~mm} \times 150 \mathrm{~mm}$, 5-Micron, Agilent, Santa Clara, CA, United States) or ultra-performance liquid chromatography (UPLC) in Agilent 1290 Infinity II (Santa Clara, CA, United States) with a linear eluting gradient $(5-40 \%$ solvent B over $7 \mathrm{~min}, 40-100 \%$ solvent B in $7-8.5 \mathrm{~min}, 100 \%$ solvent $B$ in $8.5-10 \mathrm{~min}$, and $100-5 \%$ solvent $B$ in $10-10.1 \mathrm{~min}$ ) with the same solvent $A$ and solvent $B$ at the flow rate of $0.4 \mathrm{ml} / \mathrm{min}$ with Eclipse Plus C18 reverse-phase column $(2.1 \mathrm{~mm} \times 100 \mathrm{~mm}, 1.8$-Micron, Agilent, Santa Clara, CA, United States). A photodiode array detector (Agilent, Santa Clara, CA, United States) was used for the detection of UV-visible absorption from 190 to $600 \mathrm{~nm}$. The quantification of flavonoids was through the peak area of the UV spectrum on HPLC. The products were also analyzed on a Xevo triple quadrupole mass spectrometer (TQ-MS, Waters, MA, United States) in a negative-ion mode, and the MS detection conditions were as follows: capillary voltage, $2.50 \mathrm{kV}$; cone voltage, $70 \mathrm{~V}$; desolvation gas flow, $650 \mathrm{~L} / \mathrm{h}$; cone gas flow, $50 \mathrm{~L} / \mathrm{h}$; collision gas flow, $0.12 \mathrm{ml} / \mathrm{min}$; collision energy, $30 \mathrm{eV}$; desolvation temperature, $350^{\circ} \mathrm{C}$; source temperature, $150^{\circ} \mathrm{C}$; and scan range, $100-1,000$ mass-to-charge ratio $(\mathrm{m} / \mathrm{z})$.

\section{Overexpression of LaPT2 in A. thaliana}

To test the in vivo function of LaPT2, it was subcloned into the binary vector pCAMBIA2300-35S-OCS between the sites of Kpn I and Sal I. Agrobacterium tumefaciens strain GV3101 clones containing pCAMBIA2300-35S-OCS-LaPT2 were used for transformation into Arabidopsis by using the floral dip method (Clough and Bent, 1998). T2 generation seeds were germinated on plates with MS containing $50 \mathrm{mg} / \mathrm{ml}$ kanamycin, and the resistant seedlings were transferred to soil to obtain homozygous T3 generation seeds.

For the flavonoid analysis, transgenic Arabidopsis was grown at $22^{\circ} \mathrm{C}$ with $16 / 8 \mathrm{~h}$ light and dark cycles. The 21 -day-old seedlings were collected, freeze-dried, and used for the flavonoid extraction by using the $80 \%$ method and detected on HPLC by using the abovementioned method.

For feeding assays, both wild-type and transgenic Arabidopsis plants (2-week-old seedlings) were grown on plates with or without $0.1 \mathrm{mM}$ kaempferol, and flavonoids were extracted with $80 \%$ aqueous acetone and further processed as described by Sasaki et al. (2008). The final methanol extracts from the wild-type and transgenic plants were analyzed on HPLC/MS by using the abovementioned method.

\section{RESULTS}

\section{Identification of a Candidate Flavonoid PT Gene LaPT2 in White Lupin}

In a previous study, we identified a PT gene LaPT1, which is responsible for the prenylation of isoflavone genistein and 2'-OH genistein at C3' position in white lupin (Shen et al., 2012). 
A

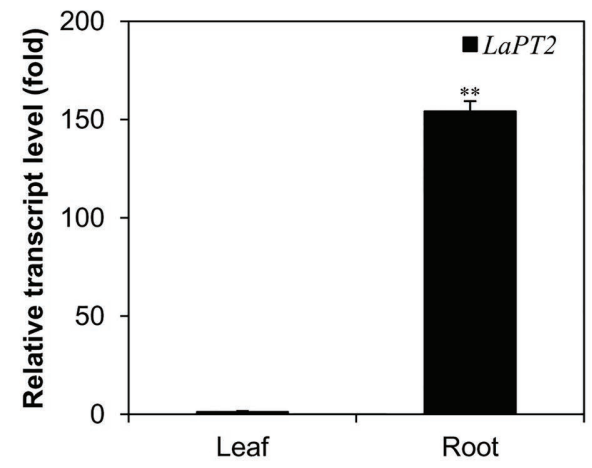

C

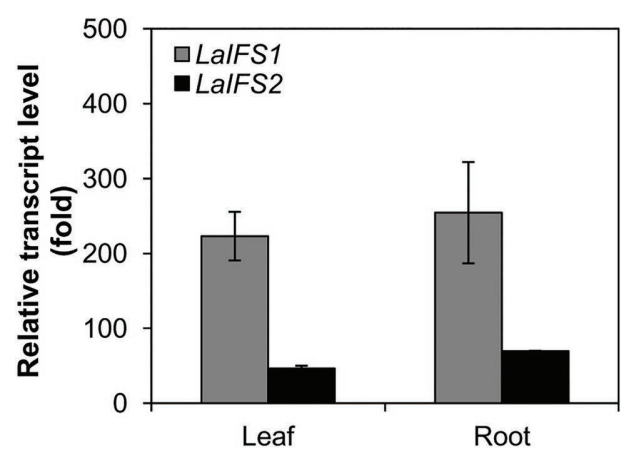

E

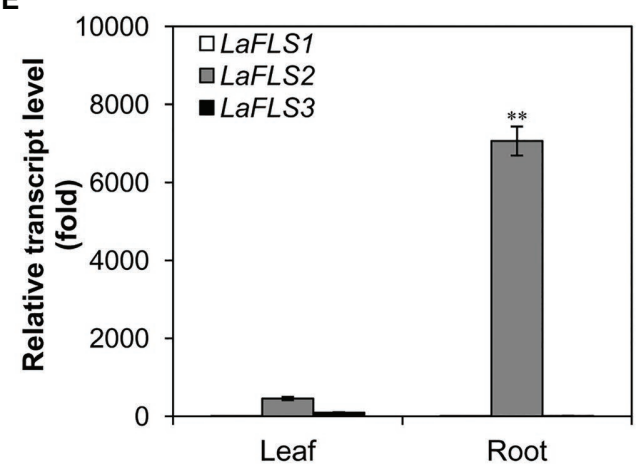

B

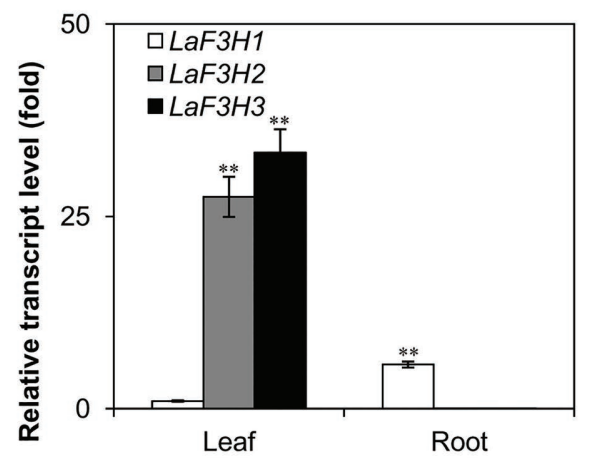

D

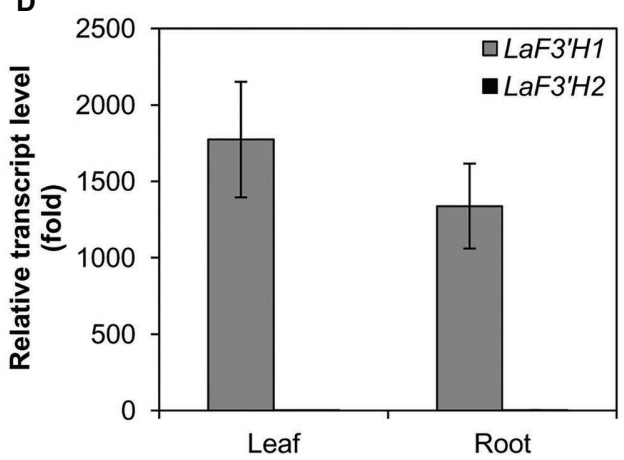

$F$

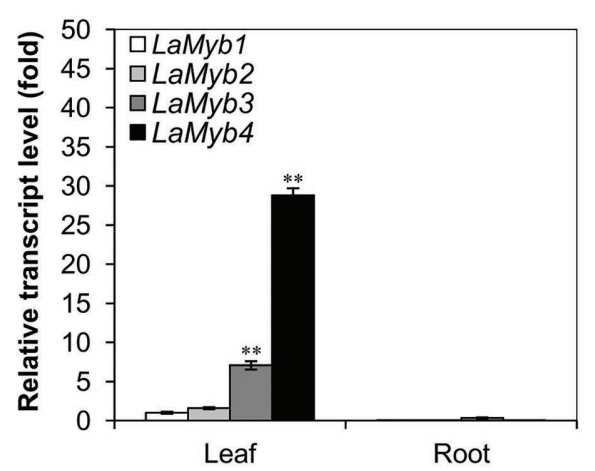

FIGURE 1 | Expression profile of LaPT2 in leaves and roots of white lupin using quantitative real-time PCR (qRT-PCR) analysis. (A-F) Relative transcript levels of LaPT2 (A), three LaF3H (B), two LalFS (C), two LaF3'H (D), three LaFLS (E), and four LaMYB (F) in leaves and roots of white lupin. In (A) the expression level of LaPT2 in leaves was set to 1 ; in $\mathbf{( B , C )}$ the expression level of $L a F 3 H 1$ in leaves was set to 1 . In (D) the expression level of $L a F 3{ }^{\prime} H 2$ in leaves was set to 1 . In (E) the expression level of LaFLS1 in leaves was set to 1. In (F) the expression level of LaMyb1 in leaves was set to 1 . Data are presented as mean \pm SD, Student's $t$-test $\left(n=3,{ }^{* *} p<0.01\right.$, comparison between leaves and roots samples for each gene) with three technical duplicates.

To further identify additional candidate genes for the biosynthesis of diverse prenylated flavonoids in white lupin, we performed a blast search with LaPT1 as a query against the white lupin transcriptome database containing 65,097 contigs (Secco et al., 2014). Two EST sequences (LAGI02_4454 and LAGI02_33083) with the highest identity (about 45\%) were obtained, and they were 1,892 and 1, $460 \mathrm{bp}$ in length, encoding deduced proteins of 407 and 402 amino acids in length, respectively.

A phylogenetic tree with other plant PTs involved in flavonoids, vitamin E, and plastoquinone biosynthesis showed that the deduced protein encoded by LAGI02_4454 was grouped within the same clade containing several flavonoid PTs from Leguminosae, whereas the deduced protein encoded by
LAGI02_33083 was clustered with PTs involved in tocopherol biosynthesis (Figure 2). Meanwhile, the deduced protein encoded by LAGI02_33083 showed a higher identity with AtVTE2-1 compared with LaPT1. Both sequence identity and phylogenetic analysis highly suggested that LAGI02_4454 was most likely to be involved in the flavonoid biosynthesis, whereas LAGI02_33083 was involved in the tocopherol biosynthesis.

Therefore, we isolated the open reading frame of LAGI02_4454 (GenBank accession No. MH298812) using RT-PCR and designated it as LaPT2. The deduced LaPT2 protein showed $59,56,49$, and $48 \%$ sequence identity with SfG8DT, SfG6DT, GuG6DT, and LaPT1, respectively, in the amino acid level. The LaPT2 protein contained two conserved PT motifs, 


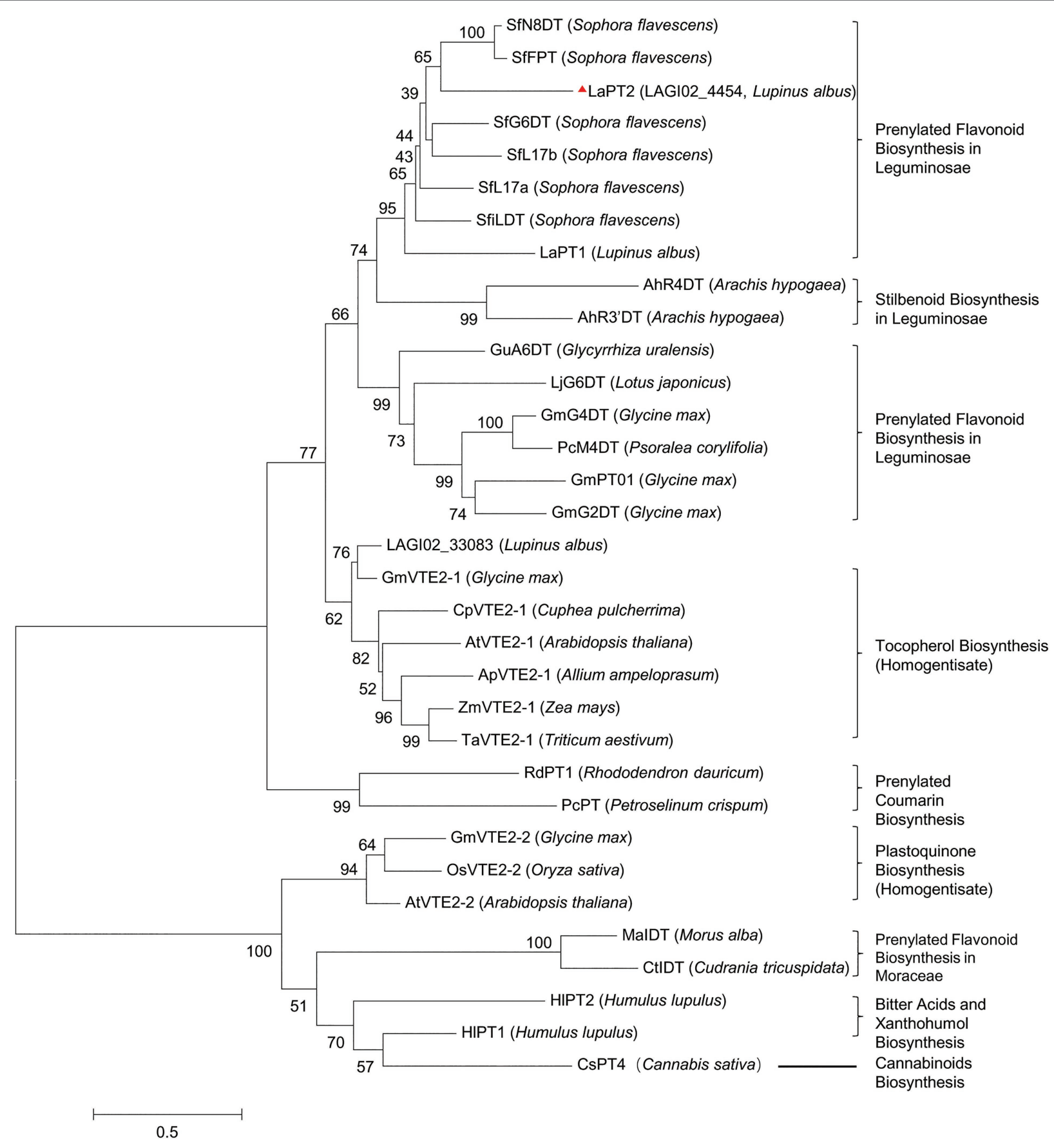

FIGURE 2 | Phylogenetic analysis of the deduced prenyltransferases (PTs) from white lupin with other closely related PTs in plant species. The multi-sequence alignment was performed by using Clustal X2. Then, the molecular phylogenetic tree based on the maximum likelihood method was reconstructed by using the MEGA6 software with a bootstrap value of 1,000. The Genbank accession number are as follows: AtVTE2-1, AY089963; ApVTE2-1, DQ231057; CpVTE2-1, DQ231058; MaIDT, KM262659; CtIDT, KM262660; AtVTE2-2, DQ231060; HIPT-1, AB543053; HIPT-2, KM222442; SfFPT, KC513505; SfN8DT-1, AB325579; SfG6DT, AB604224; SfiLDT, AB604223; LaPT1, JN228254; GmG2DT, LC140930; GmPT01, KRH76147.1; GmG4DT, AB434690; GuA6DT, KJ123716; LjG6DT, KX228696; SfL17a, AB371287; SfL17b, AB370329; LaPT2, MH298812; AhR4DT, AQM74172.1; AhR3'DT, AQM74173.1; PcM4DT, AYV64464.1; PcPT, WOSKS3.1; RdPT1, BBD96134.1; GmVTE2-2, ABB70128.1; OsVTE2-2, BAC83059.1; OsVTE2-2, BAC83059.1; ZmVTE2-1, ABB70122.1; TaVTE2-1, ABB70123.1; GmVTE2-1, ABB70126.1; and CsPT4, DAC76710.1.

NQxxDxxxD and $\mathrm{KD}(\mathrm{I} / \mathrm{L}) \mathrm{xDx}(\mathrm{E} / \mathrm{D})$, which were shared by other flavonoid PTs (Supplementary Figure 1). LaPT2 was predicted to possess seven putative transmembrane domains
(Supplementary Figure 1), which was similar to the other characterized plant flavonoid PTs with seven to nine transmembrane domains (Supplementary Figure 1). 


\section{In vitro Function of the Recombinant LaPT2 Protein}

In order to test the in vitro function of the recombinant LaPT2 protein, its open reading frame was cloned into the yeast expression vector pDR196GW. The plasmid pDR196GW-LaPT2 was transformed into the yeast strain W303A1. The extracted microsomal proteins containing LaPT2 were initially incubated with $\mathrm{Mg}^{2+}$ as a cofactor and eight representative flavonoid aglycones as the potential substrates (genistein, 2'-hydroxygenistein, daidzein, naringenin, liquiritigenin, isoliquiritigenin, apigenin, and kaempferol).

It was revealed that the recombinant LaPT2 protein could not accept genistein, 2'-hydroxygenistein, daidzein, liquiritigenin, isoliquiritigenin, or apigenin as the substrate, but showed an activity toward kaempferol and naringenin (Figure 3; Supplementary Figure 2). New products were detected by using HPLC in the different reactions with kaempferol and naringenin as a substrate (Figure 3A, left; Supplementary Figure 2A), in comparison with the control reactions (Figure $\mathbf{3 A}$, middle; Supplementary Figure 2B). The new enzymatic products with naringenin as a substrate were confirmed to be a prenylated flavonoid naringenin with monoisotopic $\mathrm{m} / \mathrm{z}$ of 339.4 by both UPLC-MS (Supplementary Figure 2C) and UPLC/MS/MS (Supplementary Figure 2D). In particular, the new enzymatic products with kaempferol as a substrate were further confirmed to be 8-prenylkaempferol (Figure 3B) as compared with the UV chromatograph (Figures 3B,C, left), MS (Figures 3B,C, middle), and MS/MS (Figures 3B,C, right) of the available authentic standard (Figure 3C), indicating that LaPT2 was able to prenylate kaempferol at the $\mathrm{C} 8$ position (Figure $3 \mathrm{D}$ ).

\section{Enzymatic Properties of the Recombinant LaPT2 Toward Other Flavonoid Substrates}

Because LaPT2 could prenylate representative flavonol of kaempferol, it could possibly also use other flavonols or additional flavonoids as substrates. Therefore, other 12 available flavonoid

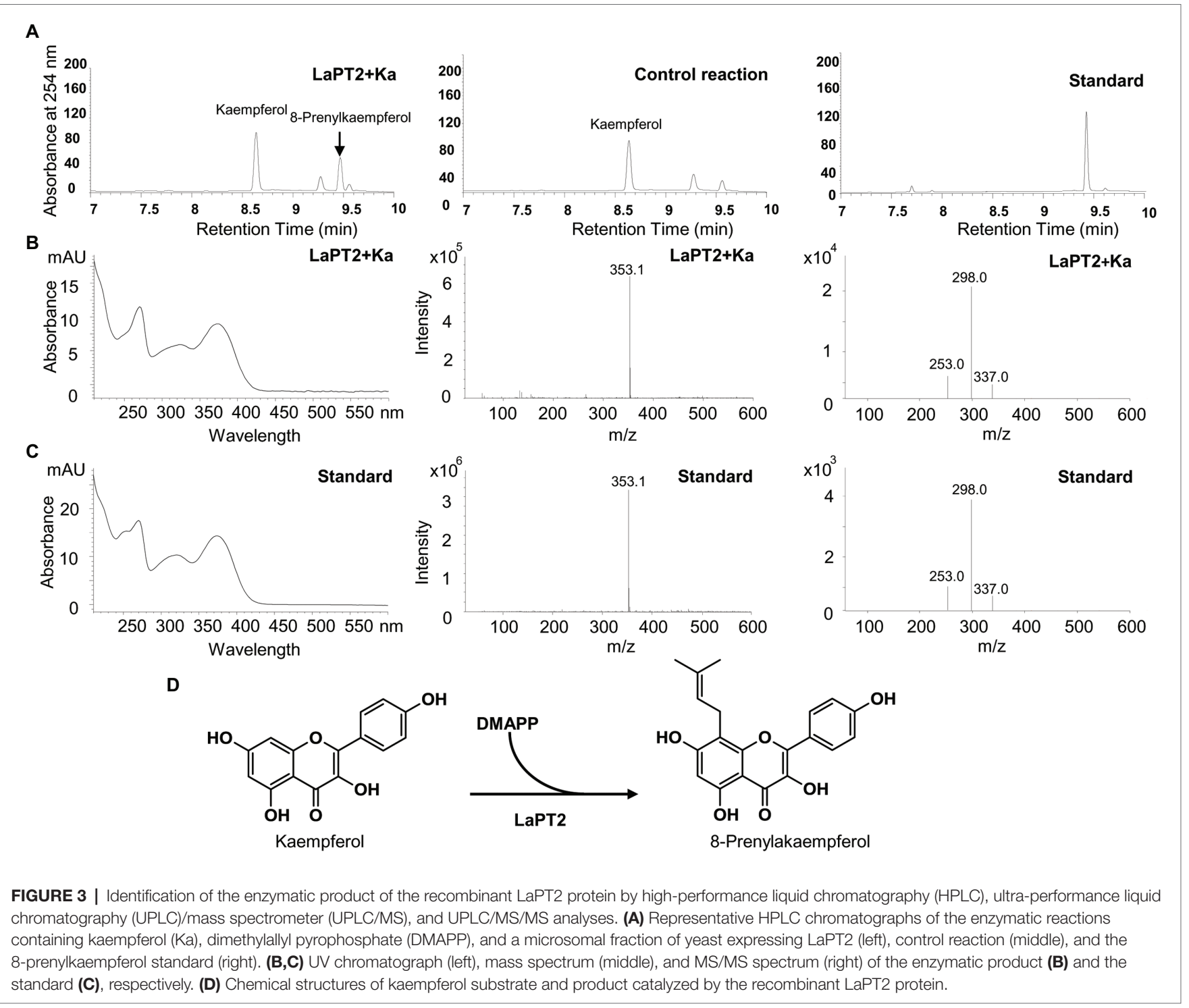


compounds, including quercetin, myricetin, morin, galangin, 5-deoxyquercetin, 3-hydroxyflavone, 4'-O-methylkaempferol, rhamnetin, kaempferol-3-O-glucoside, aromadendrin, taxifolin, and catechin, were further tested as potential substrates (Figure 4A).

It was revealed that the recombinant LaPT2 protein could use quercetin, myricetin, and morin as substrates, and they all have hydroxyl groups at $\mathrm{C} 3, \mathrm{C} 5, \mathrm{C} 7$, and $\mathrm{C} 4$ ' positions (Figure 4A; Supplementary Figures 3A-C). In addition, the recombinant LaPT2 protein could convert galangin (hydroxy group at C3, C5, and C7 positions), 5-deoxyquercetin (hydroxy group at $\mathrm{C} 3, \mathrm{C} 7, \mathrm{C}^{\prime}$, and $\mathrm{C}^{\prime}$ positions), and 4'-O-methylkaempferol (hydroxy group at C3, C5, and C7 positions) to the corresponding prenylated products (Figure 4A; Supplementary Figures 3D-F). However, 3-hydroxy flavone (without hydroxyl groups at either $\mathrm{C} 5$ and $\mathrm{C} 7$ or $\mathrm{C} 4$ ' positions) and rhamnetin (a methoxy group is substituted at $\mathrm{C} 7$ position of quercetin) could not be used as a substrate (Figure 4A), implying that the $\mathrm{OH}$ group at the $\mathrm{C} 7$ position is critical with the $\mathrm{OH}$ groups at $\mathrm{C} 3$ and $\mathrm{C} 5$ being important but not essential.

As mentioned above, LaPT2 is not active toward apigenin that lacks $\mathrm{OH}$ group at $\mathrm{C} 3$ position, but it was active toward naringenin that also lacks $\mathrm{OH}$ group at $\mathrm{C} 3$ position. However, kaempferol-3-O-glucoside (an $\mathrm{O}$-glycosyl group at $\mathrm{C} 3$ position) could not be prenylated like kaempferol (Figure 4); therefore, these data together suggested that the $\mathrm{OH}$ group at $\mathrm{C} 3$ position is critical at least for flavonols. In addition, we also found that two dihydro flavonols, aromadendrin, and taxifolin could be prenylated (Supplementary Figures 3G,H), but another structurally similar flavanol compound, catechin, could not be accepted as a substrate (Figure 4A).

The relative conversion rates of LaPT2 toward flavonoid compounds were in the order of kaempferol $(98.25 \%)>$ quercetin $(94.85 \%)>$ 5-deoxyquercetin $(51.46 \%)>$ galangin $(34.10 \%)>$ myricetin $(13.16 \%)>4$ '-O-methylkaempferol $(10.59 \%)>$ naringenin $(8.76 \%)>$ morin $(6.41 \%)>$ aromadendrin $(3.29 \%)>$ taxifolin (1.27\%; Figure 4B), implying kaempferol and quercetin, without any additional glucosylation or methylation modification, are the preferred substrates for LaPT2. Furthermore, the C2-C3 double bond is important for LaPT2 to accept the flavonols better than the other flavonoid skeletons.

In addition, the apparent $K m$ values of kaempferol, 4'-O-methylkaempferol, and quercetin were revealed to be $74.07 \pm 3.78,115.19 \pm 34.25$, and $102.81 \pm 6.41 \mu \mathrm{M}$, respectively, and they were higher than those of galangin, 5 -deoxyquercetin, morin, and aromadendrin, with the apparent $\mathrm{Km}$ values of $20.66 \pm 2.91,10.02 \pm 3.19,20.85 \pm 3.79$, and $20.08 \pm 4.44 \mu \mathrm{M}$ (Figure 4C). This result implied that kaempferol, 4'-O-methylkaempferol, and quercetin showed less affinity toward LaPT2 than galangin, 5-deoxyquercetin, morin, or aromadendrin as far as their apparent $\mathrm{Km}$ values were concerned.

In addition to DMAPP, we also detected FPP and GPP as the prenyl donor with kaempferol as a substrate. It was revealed that neither FPP nor GPP could be accepted as a prenyl donor (Supplementary Figures 4A,B), but only DMAPP with an apparent $\mathrm{Km}$ value of $66.11 \pm 7.54 \mu \mathrm{M}$. Taken together, it is clear that LaPT2 is a flavonoid PT with DMAPP as a preferred prenyl donor and flavonols as the preferred substrates.

\section{Subcellular Localization of LaPT2}

The LaPT2 protein contains a putative transit peptide of 87 amino acids at its $\mathrm{N}$-terminus for targeting the chloroplast as predicted by ChloroP 1.1. To verify its subcellular localization, the full-length LaPT2 protein fused with GFP (LaPT2-GFP), its 87-amino-acid transit peptide fused with GFP ( $\left.\mathrm{LaPT}_{1-87}-\mathrm{GFP}\right)$, and the truncated protein fused with GFP (LaPT2 ${ }_{88-403}-\mathrm{GFP}$, lacking the transit peptide) were, respectively, introduced into Arabidopsis protoplasts for the subcellular localization analysis.

Green fluorescence of the native LaPT2-GFP fusion protein was detected in protoplasts, which was overlapped with the red autofluorescence of the chloroplasts (Figure 5A). In addition, a strong green fluorescence of LaPT $2_{1-87}-\mathrm{GFP}$ was clearly observed in the chloroplasts, which was the same as for LaPT2-GFP (Figures 5A,B). In contrast, green fluorescence of the LaPT2 ${ }_{88}$ ${ }_{403}$-GFP fusion protein without the signal peptide was not found in the chloroplasts (Figure 5C), which was similar to that of the free GFP control (Figure 5D). These results clearly indicated that LaPT2 is localized to the chloroplasts, and the 87-aminoacid signal peptide is essential and in itself alone could be targeted to the correct/valid subcellular organelle.

\section{Expression Pattern of the LaPT2 Gene}

To further elucidate the expression pattern of LaPT2 in white lupin, its expression levels in leaves and roots were determined by using qRT-PCR, along with several other genes involved in the flavonol and isoflavone biosynthetic pathway. It was shown that the LaPT2 gene was preferentially expressed in roots than in leaves (Figure 1A).

Meanwhile, the expression levels of several other pathway genes were also determined, including three flavanone 3-hydroxylase (F3H; LaF3H1, 2, and 3), two 2-hydroxyisoflavanone synthases (IFS; LaIFS1 and 2), two F3'H (LaF3'H1 and 2), three flavonol synthases (FLS; LaF3H1, 2, and 3), and four $M y b$ transcription factor genes (LaMyb1, 2, 3, and 4 that showed a relatively high similarity to the MYBs involved in the flavonoid pathway from the other plant species; Figures 1B-F). In the present study, their relative expression levels in leaves and roots were compared with the gene-specific primers (Figures 1B-F; Supplementary Table 2). Some of them were expressed with a relatively high level, including LaFLS2 in roots (Figure 1E), La F3'H1 and LaIFS1 in both leaves and roots (Figures 1C,D). Among all these genes, only LaF3H1 and LaFLS2 showed the same expression pattern as LaPT2, with relatively higher expression levels in roots than in leaves, although the expression level of $L a F 3 H 1$ is lower than that of LaFLS2 (Figures 1B,E). This result implied that LaPT2 likely co-express with LaF3H1 and LaFLS2 for the production of prenylated flavonols in the roots of white lupin.

\section{Flavonoid Profiles in White Lupin}

To detect the potential presence of prenylated flavonols in white lupin, the leaves and roots of a 30-day-old white lupin were harvested to profile flavonoid compounds by using the HPLC, the UPLC-MS, and the UPLC-MS-MS analysis. In total, 11 peaks corresponding to the 12 compounds (Figures 6A,B; Supplementary Table 1) were identified, supported by UV 
A<smiles>O=c1c(O)c(-c2ccc(O)cc2)oc2cc(O)cc(O)c12</smiles><smiles>O=c1c(O)c(-c2ccc(O)cc2O)oc2cc(O)cc(O)c12</smiles>

Morin<smiles>O=c1c(O)c(-c2ccccc2)oc2ccccc12</smiles>

3-Hydroxyflavone<smiles>O=c1c(O)c(-c2ccccc2)oc2cc(O)cc(O)c12</smiles><smiles></smiles>

Quercetin<smiles>NC(=O)c1c(O)cc(O)cc1Oc1cc(O)cc(O)c1O</smiles>

Myricetin<smiles>COc1ccc(-c2oc3cc(O)cc(O)c3c(=O)c2O)cc1</smiles><smiles>O=c1c(O)c(-c2ccc(O)c(O)c2)oc2cc(O)ccc12</smiles>

5-deoxyquercetin

\section{4'-O-methylkaempferol}<smiles>COc1cc(O)c2c(=O)c(O)c(-c3ccc(O)c(O)c3)oc2c1</smiles><smiles>COc1c(-c2ccc(O)cc2)oc2cc(O)cc(O)c2c1=O</smiles>

Rhamnetin kaempferol-3-O-glucoside<smiles>O=C1c2c(O)cc(O)cc2OC(c2ccc(O)cc2)C1O</smiles>

Aromadendrin<smiles>O=C1c2c(O)cc(O)cc2OC(C2=CC=C(O)[Se](O)=C2)C1O</smiles>

Taxifolin<smiles>Oc1cc(O)c2c(c1)OC(c1ccc(O)c(O)c1)C(O)C2</smiles>

Catechin<smiles>O=C1CC(c2ccc(O)cc2)Oc2cc(O)cc(O)c21</smiles>

Naringenin

B

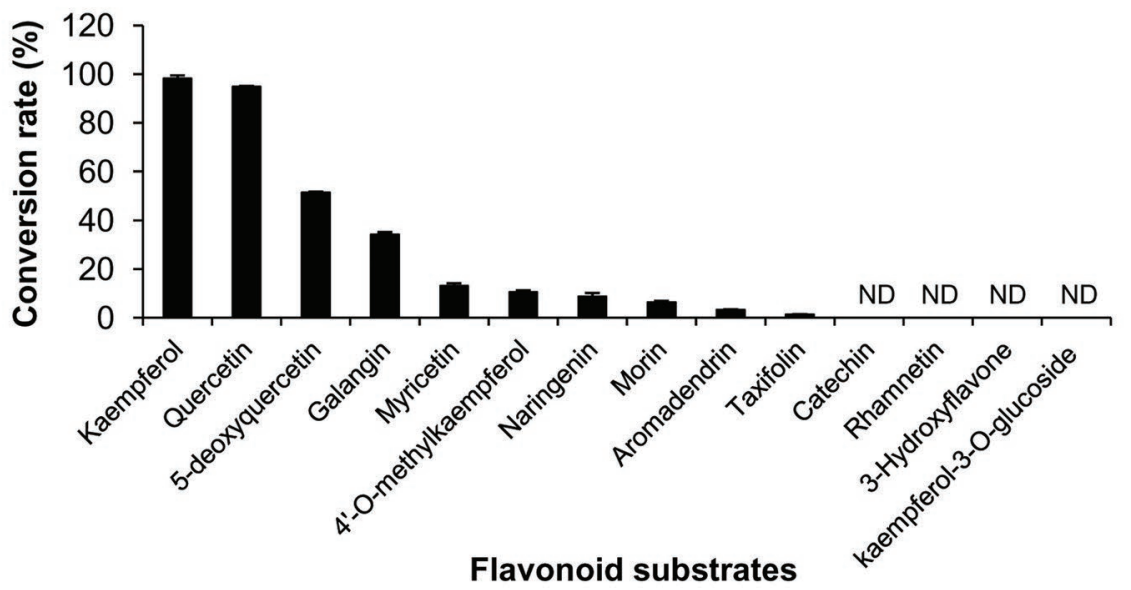

C

\begin{tabular}{lcc}
\hline Flavonoid substrates & $\boldsymbol{m} / \mathbf{z}(\mathbf{N I})$ & $\mathbf{K m}(\boldsymbol{\mu} \mathbf{M})$ \\
\hline Naringenin & 339.4 & $32.56 \pm 4.82$ \\
Kaempferol & 353.2 & $74.07 \pm 3.78$ \\
4'-O-methylkaempferol & 367.3 & $115.19 \pm 34.25$ \\
Quercetin & 369.2 & $102.81 \pm 6.41$ \\
Myricetin & $387.3(\mathrm{PI})$ & $/$ \\
Galangin & 337.2 & $20.66 \pm 2.91$ \\
5-deoxyquercetin & 353.2 & $10.02 \pm 3.19$ \\
Morin & 369.2 & $20.85 \pm 3.79$ \\
Aromadendrin & 355.1 & $20.08 \pm 4.44$ \\
Taxifolin & 371.1 & $/$ \\
3-Hydroxyflavone & N.D. & $/$ \\
Kaempferol-3-O-glucoside & N.D. & $/$ \\
Rhamnetin & N.D. & $/$ \\
Catechin & N.D. & $/$ \\
\hline
\end{tabular}

FIGURE 4 | Comparison of the enzymatic efficiency of the recombinant LaPT2 protein toward different flavonoid substrates. (A) Chemical structures of the representative flavonoid substrates tested in the present study. (B) The conversion rate of the recombinant LaPT2 protein toward different flavonoid substrates. (C) Apparent $\mathrm{Km}$ values of the recombinant LaPT2 protein toward different flavonoid substrates. $\mathrm{m} / \mathrm{z}$ is for mass-to-charge ratio, $\mathrm{NI}$ is for negative-ion mode, and PI is for positive-ion mode. The enzymatic assays were performed with technical triplicates in (B,C). 


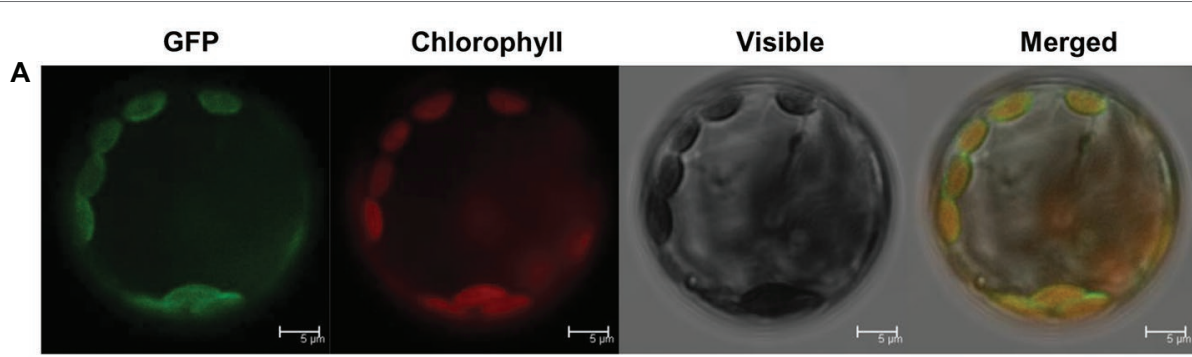

LaPT2-GFP

B

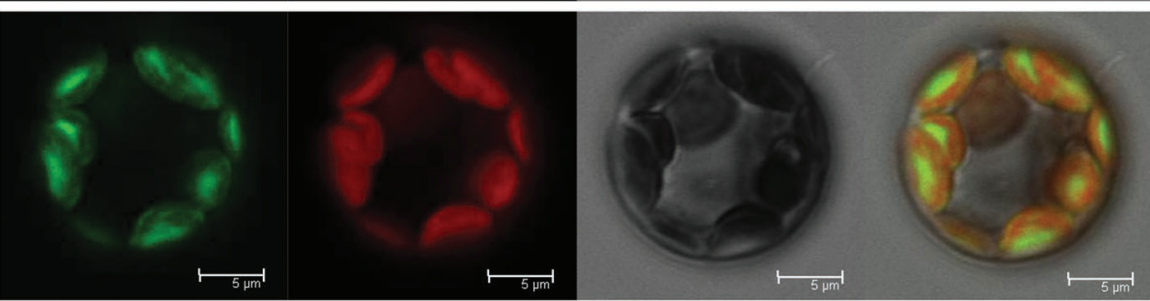

LaPT2-TP $_{1-87}$-GFP

C

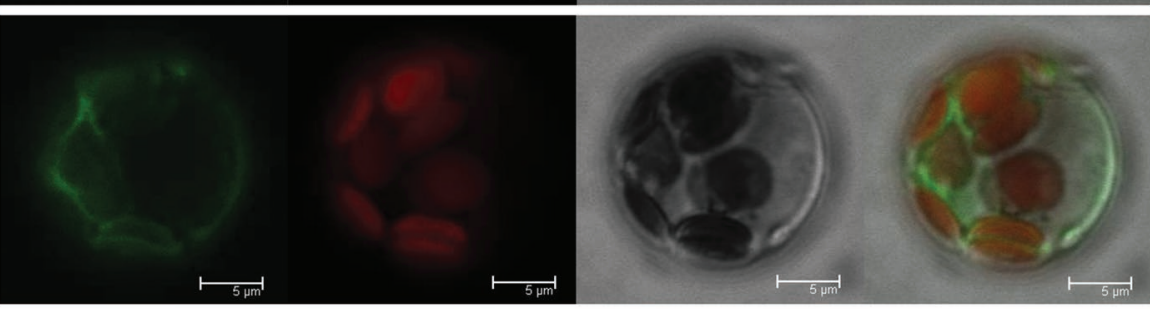

LaPT2-TP $_{88-403}$-GFP
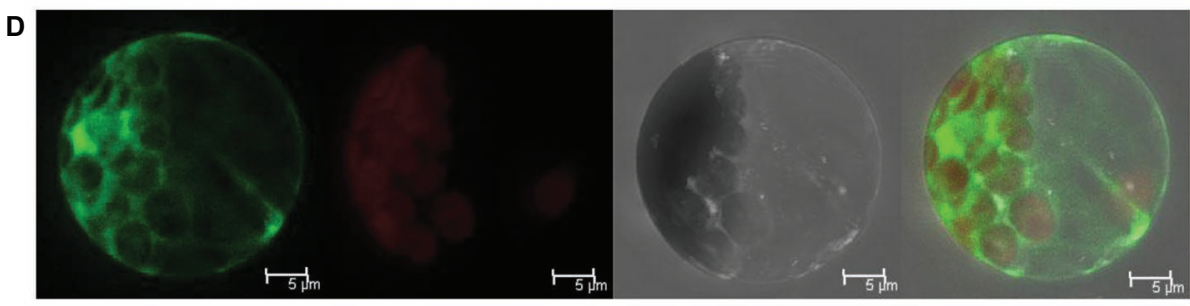

PCAMBIA1302-GFP

FIGURE 5 | Subcellular localization of the LaPT2 protein in Arabidopsis protoplasts. (A) Fluorescence signals from the protoplast transiently expressing the LaPT2green fluorescent protein (GFP) construct were observed under laser confocal scanning microscopy. Bars $=5 \mu \mathrm{m}$. (B) Fluorescence signals from the protoplast transiently expressing the LaPT2 ${ }_{1-87}$-GFP construct, which encoded only the transit peptide sequence of LaPT2. Bars $=5 \mu \mathrm{m}$. (C) Fluorescence signals from the protoplast transiently expressing the LaPT2 88 -403 -GFP construct, which lacked the transit peptide sequence of LaPT2. Bars $=5 \mu$ m. (D) Fluorescence signals from the protoplast transiently expressing pCAMBIA1302-GFP as control. Bars $=5 \mu \mathrm{m}$.

chromatographs (Supplementary Figure 5), mass spectrum (Supplementary Figure 6), and tandem mass spectrum (Supplementary Figure 7), and referred with a previous report (D'Agostina et al., 2008). Among them, seven isoflavonoids and five flavonol conjugates were detected in the leaves and/ or roots (Supplementary Table 1). In particular, isorhamnetin 3-O-galactoside (peak 6 in Figure 6A), kaempferol 3-O-glucoside (peak 4 in Figure 6A), and 2'-hydroxygenistein 4'-O-glucoside (peak 2 in Figure 6A) were relatively abundant in the leaves whereas 2'-hydroxygenistein 4'-O-glucoside (peak 2 in Figure 6B) and genistein 7-O-glucoside (peak 3 in Figure 6B) were relatively abundant in the roots (Supplementary Table 1). However, isorhamnetin 3-O-galactoside and kaempferol 3-O-glucoside were absent in the roots although they were relatively abundant in the leaves (Figure 6A; Supplementary Table 1).

In particular, it was found that a peak at the same retention time for the 8-prenylkaempferol standard was identified only in the roots (Figure 6B) but not in the leaves, although it was buried with another putative compound $(\mathrm{m} / \mathrm{z}$ 351.1) that showed different UV chromatographs, MS spectrums, and MS/ MS spectrums (Supplementary Figures 5-7 for peak 10). The peak in the roots for 8-prenylkaempferol was very small, which indicates a trace amount, but the ion fragment at the same retention times was the same as the authentic standard (m/z 353.1; Supplementary Figure 8). Taken together, the presence of 8-prenylkaempferol in the roots was confirmed by comparison with the authentic standard using the same UV chromatograph, MS, and MS/MS spectrum (Figure 6B; Supplementary Figure 8).

\section{Overexpression of LaPT2 in A. thaliana}

To verify the in vivo function of LaPT2, it was overexpressed in A. thaliana that is rich in flavonol kaempferol in the leaves. Three independent lines that showed high expression levels of LaPT2, which were confirmed by using qRT-PCR (Supplementary Figure 9A), and the flavonoid profiles in the 


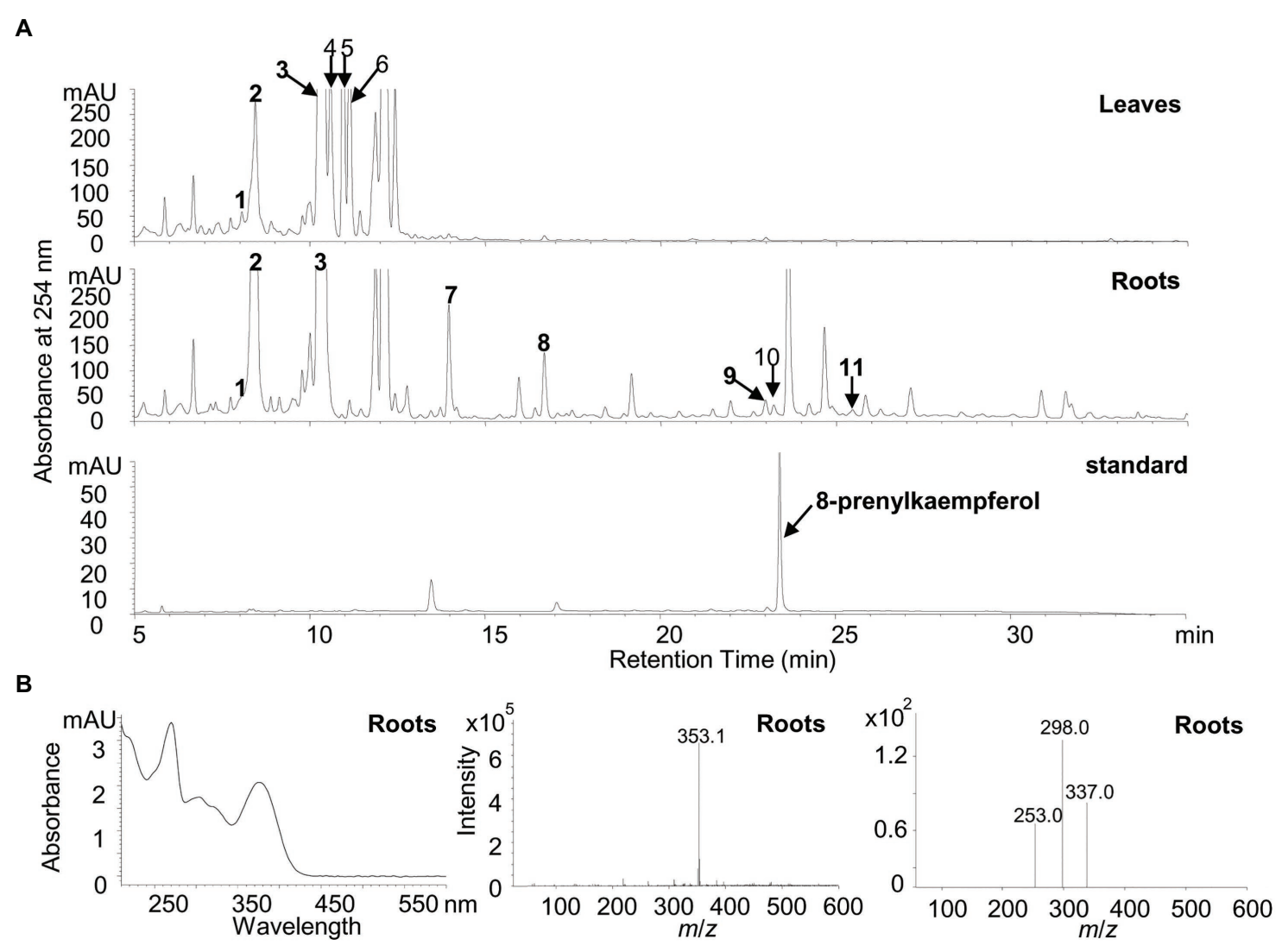

FIGURE 6 | Identification of putative flavonoid compounds in leaves and roots of white lupin using HPLC, UPLC/MS, and UPLC/MS/MS. (A) Representative HPLC chromatographs of the methanolic extracts from white lupin leaves (upper panel), roots (middle panel), and the 8-prenylkaempferol standard (lower panel). Numbers indicated identified flavonoids as shown in Supplementary Table 1. (B) UV chromatograph (left), mass spectrum (middle), and tandem mass spectrum (right) of 8-prenylkaempferol identified in roots.

seedlings were analyzed on HPLC. No significant difference or distinct new peaks were detected in the transgenic lines in comparison with the wild type and the 8-prenylkaempferol standard (Supplementary Figure 9B). In addition, we further fed the seedlings with kaempferol on agar plates and detected the flavonoid profiles using the UPLC/MS. However, only the level of kaempferol 3-O-glucoside-7-O-rhamnoside (K3G7R) was increased in both transgenic and the wild-type lines (Supplementary Figures 9C,D), no additional new peak/fragment was detected by searching a targeted ion fragment of 353 for prenylkaempferol.

\section{DISCUSSION}

Several prenylated flavonoid compounds have been isolated from the different plant species, especially in Leguminosae. Some legume plant species accumulate prenylated flavonoids, such as gluceollins in soybean (Schmidt et al., 1992) and wighteone in L. japonicus (Liu et al., 2018), under environmental stress, whereas some plants constitutively produce prenylated flavonoids under the normal growth condition, as in white lupin (Tahara et al., 1984; Gagnon et al., 1992; Katagiri et al., 2000). In the present study, we identified another PT gene LaPT2 in white lupin and found that it encodes a putative flavonoid PT as revealed by both sequence and phylogenetic analyses (Figure 2; Supplementary Figure 1). The deduced LaPT2 protein was localized in the chloroplast (Figure 5), which is the same as for the other characterized flavonoid PTs (Sasaki et al., 2008; Akashi et al., 2009; Shen et al., 2012). Further study on subcellular localization confirmed that the transit peptide of LaPT2 carries the localization signal, and the deletion of the $\mathrm{N}$-terminus signal peptide sequence leads to an incorrect localization (Figure 5), which is the same as for the other PT (Sasaki et al., 2008; Akashi et al., 2009; Shen et al., 2012; Liu et al., 2018), and all these studies demonstrated that the presence of a signal peptide is essential for the correct localization of PT proteins.

\section{Flavonoid Accumulation and the Expression Level of Related Pathway Genes}

Legume plants produce a large number of flavonoid compounds, which are the result of complex biosynthesis and regulation. Our present study showed that white lupin accumulates both isoflavonoid and flavonol conjugates in leaves and roots (Figure 6). We found that the isoflavonoid and flavonol accumulation pattern was associated with the expression 
pattern of the corresponding key pathway genes (Figures 1, 6, 7). The relatively higher expression levels of LaIFS1 in both leaves and roots may explain the abundance of glucosides of genistein and 2'-hydroxygenistein in both leaves and roots (Figures 1C, 6A,B; Supplementary Table 1). Although $\mathrm{LaF3} \mathrm{H}^{\prime} \mathrm{H}$ was highly expressed in both leaves and roots, the quercetin conjugate isorhamnetin 3-O-galactoside was accumulated at a high level in leaves but not in roots (Figures 1D, 6A,B; Supplementary Table 1), indicating that the other genes rather than LaF3'H1 might be a key gene for the biosynthesis of isorhamnetin 3-O-galactoside and other quercetin conjugates in white lupin. Although four $M y b$ genes were detected, their relative expression levels were not high in comparison with other structural genes, indicating that they may not be the key genes for the flavonoid accumulation in white lupin.

In addition, the prenylated compounds (luteone, wighteone, and prenylkaempferol) in white lupin were detected and showed relatively high levels in roots than in leaves, in the current and in the previous study (D'Agostina et al., 2008), which is associated with a relatively high level of expression of LaPT2 genes in this study and the LaPT1 gene in the previous study (Shen et al., 2012). It was clear that roots might be a desirable tissue for further discovery and identification of additional flavonoid PT genes in white lupin. Meanwhile, except 8-prenylkaempferol, the prenylated products of other flavonoids identified in in vitro enzymatic assays for LaPT2 were not discovered in a white lupin plant, and the inconsistence behavior of in vitro and in vivo activity was also observed for the PTs identified in other plant species like S. flavescens (Yazaki et al., 2009; Chen et al., 2013). In another aspect, LaFLS2 coexpressed with LaPT2 in the roots; therefore, it might be more ideal to produce a prenylated flavonoid by combining structural genes (e.g., LaFLS2 and LaPT2) in vitro for commercial purposes.

On the other hand, leaves of the wild type A. thaliana accumulate mainly kaempferol conjugates, indicating the presence of kaempferol, but no prenylated kaempferol was detected when LaPT2 was overexpressed. Even when kaempferol was fed into the transgenic A. thaliana, no additional prenylated kaempferol was produced (Supplementary Figure 9). This result was consistent with the fact that kaempferol glucosides could not be prenylated in the in vitro enzymatic assays owing to preferential modification of kaempferol as glucosides or rhamnosides in the leaves of $A$. thaliana, whereas LaPT2 is expected to act in roots. In addition, the feeding of kaempferol in $A$. thaliana has led to a more metabolic flux to K3G7R (Supplementary Figure 9), which may be explained by the competition between the prenylation and glycosylation of flavonoids in planta. Recent studies on Artocarpus heterophyllus showed that the substrate specificity of flavonoid PTs AhPT1 may change with a different cofactor, i.e., $\mathrm{Mn}^{2+}$ instead of $\mathrm{Mg}^{2+}$. As $\mathrm{Mn}^{2+}$ is an essential cofactor for photosynthetic activity, it might be accumulated in the chloroplast exposed to light and to which LaPT2 might be also targeted (Yang et al., 2020). In addition to these, the other factors may also affect the in vivo enzymatic activity of LaPT2 in ectopic plant species; therefore, the in vivo function and properties of LaPT2 protein require further investigation in the near future.

\section{LaPT2 Preferred Flavonol Aglycones as Substrates}

The LaPT2 protein showed the closest relationship with SfG6DT and LaPT1 in the phylogenetic tree, and white lupin accumulates a significant amount of prenylated isoflavonoids, but LaPT2

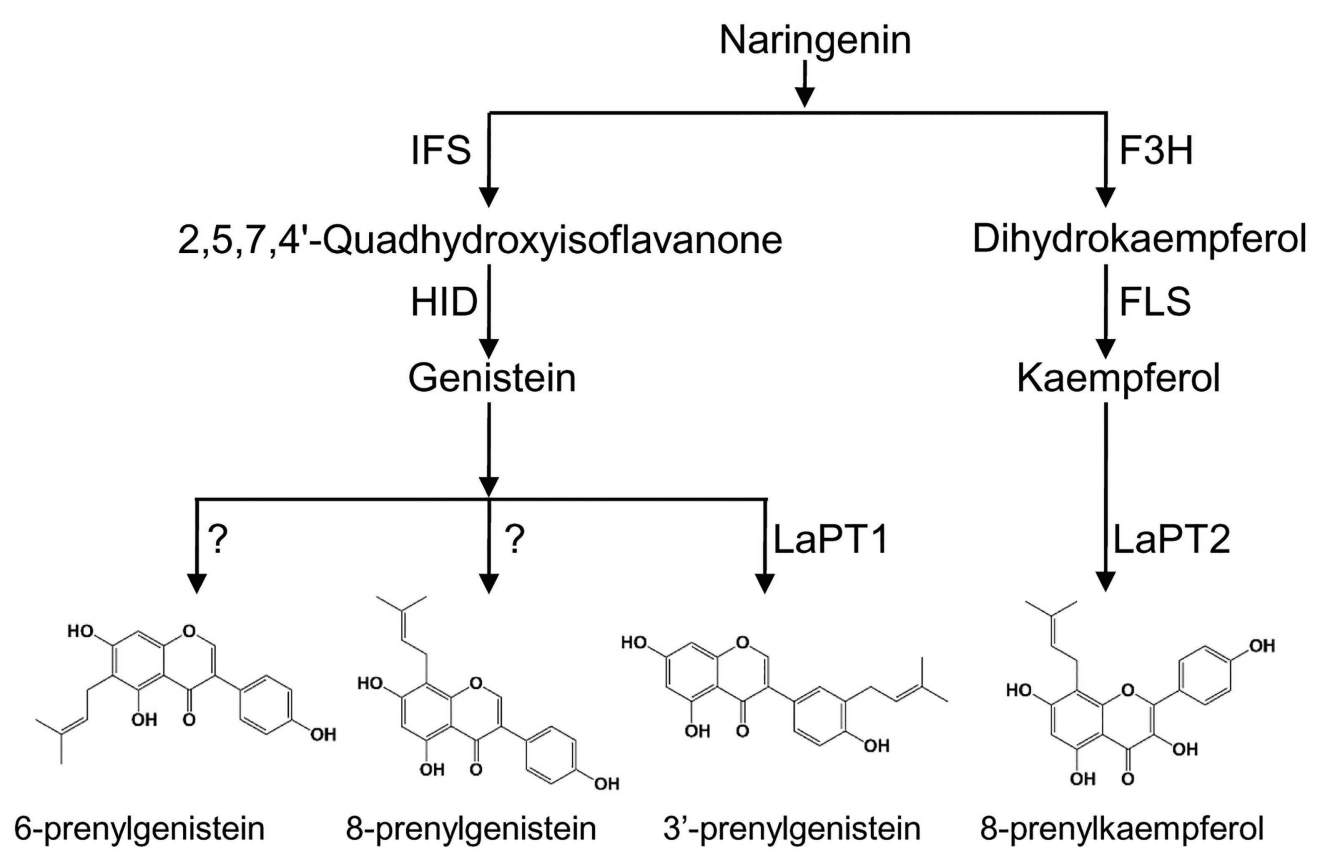

FIGURE 7 | Proposed biosynthetic pathway of prenylated flavonoids in white lupin. F3H, flavanone 3-hydroxylase; FLS, flavonol synthase; IFS, 2-hydroxyisoflavanone synthase; HID, 2-hydroxyisoflavanone dehydratase; and PT, prenyltransferase. 
exhibited no enzymatic activity toward isoflavones, which is different from SfG6DT and LaPT1. The results indicated that LaPT2 was able to catalyze the prenylation of flavonol kaempferol, and flavanone naringenin, which is the same as for SfN8DT (Sasaki et al., 2008). However, LaPT2 displayed a higher conversion rate toward kaempferol than naringenin, which was different from SfN8DT that was more active toward naringenin than kaempferol (Sasaki et al., 2008). With the presence of prenylated flavonoid kaempferol in white lupin (even though it was in trace amount and buried with another putative compound) and the high conversion rate of LaPT2 toward kaempferol, it is reasonable to speculate that kaempferol serves as the natural substrate for LaPT2 in white lupin.

Most flavonoid PTs from legume plants displayed strictly the substrate specificity (Akashi et al., 2009; Sasaki et al., 2011; Shen et al., 2012; Liu et al., 2018). Among all flavonoid-specific PTs described previously, only GuA6DT from G. uralensis and Sophora flavescens flavonoid prenyltransferase (SfFPT) from $S$. flavescens displayed relatively broad substrate specificity toward flavonoid compounds with a similar structure (Chen et al., 2013; Li et al., 2014). In our study, the recombinant LaPT2 protein showed a catalytic activity toward more flavonoltype than the other types of flavonoids (Figure 4). The fact that LaPT2 did not catalyze 3-hydroxy flavone indicated that one and/or more hydroxyl groups at C4', C5, and C7 positions of the flavonol skeleton were critical for the recognition of prenylation substrates. Moreover, the double bond between C2 and $\mathrm{C} 3$ is important for LaPT2 to accept flavonols as preferred substrates than the other flavonoid skeletons.

Many flavonol aglycones are modified through the addition of hydroxyl and/or methoxy groups on the B-ring based on the skeletal structure of kaempferol. Although LaPT2 is active toward various flavonol compounds, the maximum conversion efficiency was observed for kaempferol under the same condition (Figure 4). The prenylation reaction catalyzed by the PT represented a Friedel-Crafts alkylation of the flavonoid skeleton in the biosynthesis of prenylflavonoids (Chen et al., 2013; Li et al., 2014). The hydroxyl groups at the C7/C5 position of A-ring and $\mathrm{C}^{\prime} / \mathrm{C}^{\prime}$ ' position of B-ring would increase prenylation efficiency of SfFPT while the methoxy group on the abovementioned positions would decrease efficiency (Chen et al., 2013). It was similar for LaPT2 that the presence of methoxy group did decrease the prenylation efficiency owing to the decrease in the conversion rate from kaempferol to 4'-O-methoylkaempferol and from quercetin to rhamnetin (no activity for rhamnetin; Figure 4) whereas the presence of hydroxyl groups at C7/C5 increased prenylation efficiency owing to the increase in the conversion rate from 5-deoxyquercein to quercetin (Figure 4). However, the hydroxyl group at C-3'/ $\mathrm{C} 4$ ' reduces the prenylation efficiency for LaPT2 as the conversion rate decreased from kaempferol, quercetin to myricetin, from galangin to morin, and from aromadendrin to taxifolin (Figure 4B), which is different from SfFPT (Chen et al., 2013).

In this study, we found that LaPT2 could not accept kaempferol-3-O-glucoside as a substrate, which was similar to SfFTP that could not prenylate glycosylated flavanone (Chen et al., 2013). Therefore, it was hypothesized that prenylation precedes glycosylation in the production of complicated flavonoid compounds, for example, the prenylation reaction precedes the glycosylation step in epimedoside, a biosynthesis in Epimedium diphyllum (Yamamoto et al., 1997). Meanwhile, glucosylation may stabilize the accumulation of transit prenylated flavonoids in a plant as the detection of trace amounts of prenylkaempferol in the roots of white lupin (Figure 6).

White lupin produces different types of prenylisoflavones (Tahara et al., 1984; D’Agostina et al., 2008), but only one PT gene LaPT1 was identified to be able to prenylate isoflavonoid $(2 ' \mathrm{OH})$ genistein at $\mathrm{C}^{\prime}$ position and LaPT2 prefers flavonols as a substrate but not isoflavonoids (Figure 7). We speculate that more flavonoid PT genes were present in the white lupin genome, besides LaPT1 and LaPT2. On the basis of the BLAST search, a few more candidate PT EST sequences with low identity to LaPT1 were also yielded, and their functions were still under further investigation for the full elucidation of the prenylation mechanism of flavonoids in legume plants.

\section{DATA AVAILABILITY STATEMENT}

The datasets presented in this study can be found in online repositories. The names of the repository/repositories and accession number(s) can be found in the article/ Supplementary Material.

\section{AUTHOR CONTRIBUTIONS}

JL, GS, and YP conceived the project and designed the experiments. JL, YX, and WJ performed the experiments and analyzed the data. JL, YX, and GS prepared the manuscript. YP revised the manuscript and supervised the project. All authors contributed to the article and approved the submitted version.

\section{FUNDING}

This work was supported by the National Nature Science Foundation of China (U1906201), the Basic Scientific Research Funding of CAAS (2019-YWF-YB-06), and the Central Publicinterest Scientific Institution Basal Research Fund (No. Y2020GH01-3).

\section{ACKNOWLEDGMENTS}

We thank Baolin Guo from the Institute of Medicinal Plant Development for providing the 8-prenylkaempferol standard.

\section{SUPPLEMENTARY MATERIAL}

The Supplementary Material for this article can be found online at: https://www.frontiersin.org/articles/10.3389/fpls.2021.673337/ full\#supplementary-material 


\section{REFERENCES}

Ahmed-Belkacem, A., Pozza, A., Munoz-Martinez, F., Bates, S. E., Castanys, S., Gamarro, F., et al. (2005). Flavonoid structure-activity studies identify 6-prenyichrysin and tectochrysin as potent and specific inhibitors of breast cancer resistance protein ABCG2. Cancer Res. 65, 4852-4860. doi: 10.1158/0008-5472.CAN-04-1817

Akashi, T., Sasaki, K., Aoki, T., Ayabe, S., and Yazaki, K. (2009). Molecular cloning and characterization of a cDNA for pterocarpan 4-dimethylallyltransferase catalyzing the key prenylation step in the biosynthesis of glyceollin, a soybean phytoalexin. Plant Physiol. 149, 683-693. doi: 10.1104/ pp. 108.123679

Barron, D., and Ibrahim, R. K. (1996). Isoprenylated flavonoids-A survey. Phytochemistry 43, 921-982. doi: 10.1016/S0031-9422(96)00344-5

Bednarek, P., Franski, R., Kerhoas, L., Einhorn, J., Wojtaszek, P., and Stobiecki, M. (2001). Profiling changes in metabolism of isoflavonoids and their conjugates in Lupinus albus treated with biotic elicitor. Phytochemistry 56, 77-85. doi: 10.1016/S0031-9422(00)00366-6

Boland, G. M., and Donnelly, D. M. X. (1998). Isoflavonoids and related compounds. Nat. Prod. Rep. 15, 241-260. doi: 10.1039/a815241y

Boozari, M., Soltani, S., and Iranshahi, M. (2019). Biologically active prenylated flavonoids from the genusSophoraand their structure-activity relationship-A review. Phytother. Res. 33, 546-560. doi: 10.1002/ptr.6265

Botta, B., Menendez, P., Zappia, G., de Lima, R. A., Torge, R., and Monachea, G. D. (2009). Prenylated isoflavonoids: botanical distribution, structures, biological activities and biotechnological studies. An update (1995-2006). Curr. Med. Chem. 16, 3414-3468. doi: 10.2174/ 092986709789057662

Botta, B., Monache, G. D., Menendez, P., and Boffi, A. (2005). Novel prenyltransferase enzymes as a tool for flavonoid prenylation. Trends Pharmacol. Sci. 26, 606-608. doi: 10.1016/j.tips.2005.09.012

Bradford, M. M. (1976). A rapid and sensitive method for the quantitation of microgram quantities of protein utilizing the principle of protein-dye binding. Anal. Biochem. 72, 248-254. doi: 10.1016/0003-2697(76)90527-3

Chen, R. D., Liu, X., Zou, J. H., Yin, Y. Z., Ou, B., Li, J. H., et al. (2013). Regio- and stereospecific prenylation of flavonoids by Sophora flavescens prenyltransferase. Adv. Synth. Catal. 355, 1817-1828. doi: 10.1002/ adsc. 201300196

Clough, S. J., and Bent, A. F. (1998). Floral dip: a simplified method for Agrobacterium-mediated transformation of Arabidopsis thaliana. Plant J. 16, 735-743. doi: 10.1046/j.1365-313x.1998.00343.x

D’Agostina, A., Boschin, G., Resta, D., Annicchiarico, P., and Arnoldi, A. (2008). Changes of isoflavones during the growth cycle of Lupinus albus. J. Agric. Food Chem. 56, 4450-4456. doi: 10.1021/jf8003724

De Naeyer, A., Vanden Berghe, W., Pocock, V., Milligan, S., Haegeman, G., and De Keukeleire, D. (2004). Estrogenic and anticarcinogenic properties of kurarinone, a lavandulyl flavanone from the roots of Sophora flavescens. J. Nat. Prod. 67, 1829-1832. doi: 10.1021/np040069a

Dixon, R. A., and Paiva, N. L. (1995). Stress-induced phenylpropanoid metabolism. Plant Cell 7, 1085-1097. doi: 10.2307/3870059

Dixon, R. A., and Pasinetti, G. M. (2010). Flavonoids and isoflavonoids: from plant biology to agriculture and neuroscience. Plant Physiol. 154, 453-457. doi: $10.1104 /$ pp.110.161430

Gagnon, H., Seguin, J., Bleichert, E., Tahara, S., and Ibrahim, R. K. (1992). Biosynthesis of white lupin isoflavonoids from $\left[\mathrm{U}-{ }^{14} \mathrm{C}\right] \mathrm{L}$-phenylalanine and their release into the culture medium. Plant Physiol. 100, 76-79. doi: 10.1104/ pp.100.1.76

Han, A. R., Kang, Y. J., Windono, T., Lee, S. K., and Seo, E. K. (2006). Prenylated flavonoids from the heartwood of Artocarpus communis with inhibitory activity on lipopolysaccharide-induced nitric oxide production. J. Nat. Prod. 69, 719-721. doi: 10.1021/np0600346

Jung, H. A., Jin, S. E., Park, J. S., and Choi, J. S. (2011). Antidiabetic complications and anti-Alzheimer activities of sophoflavescenol, a prenylated flavonol from Sophora flavescens, and its structure-activity relationship. Phytother. Res. 25, 709-715. doi: $10.1002 /$ ptr.3326

Katagiri, Y., Ibrahim, R. K., and Tahara, S. (2000). HPLC analysis of white lupin isoflavonoids. Biosci. Biotechnol. Biochem. 64, 1118-1125. doi: 10.1271/ bbb. 64.1118
Larkin, M. A., Blackshields, G., Brown, N. P., Chenna, R., McGettigan, P. A., McWilliam, H., et al. (2007). Clustal W and Clustal X version 2.0. Bioinformatics 23, 2947-2948. doi: 10.1093/bioinformatics/btm404

Li, J., Chen, R., Wang, R., Liu, X., Xie, K., Chen, D., et al. (2018). Biocatalytic access to diverse prenylflavonoids by combining a regiospecific C-prenyltransferase and a stereospecific chalcone isomerase. Acta Pharm. Sin. B 8, 678-686. doi: 10.1016/j.apsb.2018.01.009

Li, J., Chen, R., Wang, R., Liu, X., Xie, D., Zou, J., et al. (2014). GuA6DT, a regiospecific prenyltransferase from Glycyrrhiza uralensis, catalyzes the 6-prenylation of flavones. ChemBioChem 15, 1673-1681. doi: 10.1002/ cbic. 201402160

Liu, J., Jiang, W., Xia, Y., Wang, X., Shen, G., and Pang, Y. (2018). Genisteinspecific G6DT gene for the inducible production of wighteone in Lotus japonicus. Plant Cell Physiol. 59, 128-141. doi: 10.1093/pcp/pcx167

Lucas, M. M., Stoddard, F. L., Annicchiarico, P., Frias, J., Martinez-Villaluenga, C., Sussmann, D., et al. (2015). The future of lupin as a protein crop in Europe. Front. Plant Sci. 6:705. doi: 10.3389/fpls.2015.00705

Middleton, E. (1998). Effect of plant flavonoids on immune and inflammatory cell function. Adv. Exp. Med. Biol. 439, 175-182.

Sasaki, K., Mito, K., Ohara, K., Yamamoto, H., and Yazaki, K. (2008). Cloning and characterization of naringenin 8-prenyltransferase, a flavonoid-specific prenyltransferase of Sophora flavescens. Plant Physiol. 146, 1075-1084. doi: 10.1104/pp.107.110544

Sasaki, K., Tsurumaru, Y., Yamamoto, H., and Yazaki, K. (2011). Molecular characterization of a membrane-bound prenyltransferase specific for isoflavone from Sophora flavescens. J. Biol. Chem. 286, 24125-24134. doi: 10.1074/jbc. M111.244426

Schmidt, P. E., Parniske, M., and Werner, D. (1992). Production of the phytoalexin glyceollin I by soybean roots in response to symbiotic and pathogenic infection. Botanica Acta 105, 18-25. doi: 10.1111/j.1438-8677.1992. tb00262.x

Secco, D., Shou, H. X., Whelan, J., and Berkowitz, O. (2014). RNA-seq analysis identifies an intricate regulatory network controlling cluster root development in white lupin. BMC Genomics 15:230. doi: 10.1186/1471-2164-15-230

Shen, G. A., Huhman, D., Lei, Z. T., Snyder, J., Sumner, L. W., and Dixon, R. A. (2012). Characterization of an isoflavonoid-specific prenyltransferase from Lupinus albus. Plant Physiol. 159, 70-80. doi: 10.1104/pp.112.195271

Sohn, H. Y., Son, K. H., Kwon, C. S., Kwon, G. S., and Kang, S. S. (2004). Antimicrobial and cytotoxic activity of 18 prenylated flavonoids isolated from medicinal plants: Morus alba L., Morus mongolica Schneider, Broussnetia papyrifera (L.) vent, Sophora flavescens ait and Echinosophora koreensis Nakai. Phytomedicine 11, 666-672. doi: 10.1016/j.phymed.2003.09.005

Sukumaran, A., McDowell, T., Chen, L., Renaud, J., and Dhaubhadel, S. (2018). Isoflavonoid-specific prenyltransferase gene family in soybean: GmPT01, a pterocarpan 2-dimethylallyltransferase involved in glyceollin biosynthesis. Plant J. 96, 966-981. doi: 10.1111/tpj.14083

Tahara, S., and Ibrahim, R. K. (1995). Prenylated isoflavonoids-an update. Phytochemistry 38, 1073-1094. doi: 10.1016/0031-9422(94)00788-U

Tahara, S., Ingham, J. L., Nakahara, S., Mizutani, J., and Harborne, J. B. (1984). Antifungal isoflavones in Lupins. Phytochemistry 23, 1889-1900. doi: 10.1016/ S0031-9422(00)84936-5

Tamura, K., Stecher, G., Peterson, D., Filipski, A., and Kumar, S. (2013). MEGA6: molecular evolutionary genetics analysis version 6.0. Mol. Biol. Evol. 30, 2725-2729. doi: 10.1093/molbev/mst197

Winkel-Shirley, B. (2001). Flavonoid biosynthesis. A colorful model for genetics, biochemistry, cell biology, and biotechnology. Plant Physiol. 126, 485-493. doi: $10.1104 / p p .126 .2 .485$

Yamamoto, H., Kimata, J., Senda, M., and Inoue, K. (1997). Dimethylallyl diphosphate: kaempferol 8-dimethylallyl transferase in Epimedium diphyllum cell suspension cultures. Phytochemistry 44, 23-28. doi: 10.1016/ S0031-9422(96)00484-0

Yamamoto, H., Senda, M., and Inoue, K. (2000). Flavanone 8-dimethylallyltransferase in Sophora flavescens cell suspension cultures. Phytochemistry 54, 649-655. doi: 10.1016/S0031-9422(00)00198-9

Yang, J., Zhou, T., Jiang, Y., and Yang, B. (2020). Substrate specificity change of a flavonoid prenyltransferase AhPT1 induced by metal ion. Int. J. Biol. Macromol. 153, 264-275. doi: 10.1016/j.ijbiomac.2020.03.005 
Yazaki, K., Sasaki, K., and Tsurumaru, Y. (2009). Prenylation of aromatic compounds, a key diversification of plant secondary metabolites. Phytochemistry 70, 1739-1745. doi: 10.1016/j.phytochem.2009.08.023

Yoneyama, K., Akashi, T., and Aoki, T. (2016). Molecular characterization of soybean pterocarpan 2-dimethylallyltransferase in glyceollin biosynthesis: local gene and whole-genome duplications of prenyltransferase genes led to the structural diversity of soybean prenylated isoflavonoids. Plant Cell Physiol. 57, 2497-2509. doi: 10.1093/pcp/pcw178

Yoo, S. D., Cho, Y. H., and Sheen, J. (2007). Arabidopsis mesophyll protoplasts: a versatile cell system for transient gene expression analysis. Nat. Protoc. 2, 1565-1572. doi: 10.1038/nprot.2007.199

Zhao, P., Inoue, K., Kouno, I., and Yamamoto, H. (2003). Characterization of leachianone G 2 “-dimethylallyltransferase," a novel prenyl side-chain elongation enzyme for the formation of the lavandulyl group of sophoraflavanone $G$ in Sophora flavescens ait. Cell suspension cultures. Plant Physiol. 133, 1306-1313. doi: 10.1104/pp.103.025213

Conflict of Interest: The authors declare that the research was conducted in the absence of any commercial or financial relationships that could be construed as a potential conflict of interest.

Copyright (C) 2021 Liu, Xia, Jiang, Shen and Pang. This is an open-access article distributed under the terms of the Creative Commons Attribution License (CC BY). The use, distribution or reproduction in other forums is permitted, provided the original author(s) and the copyright owner(s) are credited and that the original publication in this journal is cited, in accordance with accepted academic practice. No use, distribution or reproduction is permitted which does not comply with these terms. 